\title{
“It's junk food and chicken nuggets”: Children's perspectives on 'kids' food' and the question of food classification
}

\section{Charlene Elliott}

Faculty of Arts, University of Calgary, 2500 University Drive NW, Calgary, Alberta T2N1N4, Canada

\begin{abstract}
Given the expansive nature of children's food, banning the advertising of poorly nutritious products to children only deals with part of the problem. What is missing is an understanding of how child-oriented food marketing has reconfigured children's broader perceptions of what food means and the kinds of foods that are 'for them'. Drawing from focus groups conducted across Canada, this article examines the perspectives of 225 children who discussed both 'kids' food' and 'adult food'. The research reveals the broader implications of particular food marketing strategies. When children think of 'kids' food', they generally think of junk food, sugar, sugary cereals and the fun shapes and unusual colours characterizing much of contemporary childoriented packaged food. When children think of 'adult food', they think of fruits, vegetables and meat. In short, 'adult foods' are generally the unprocessed fruits, vegetables and meats that all North Americans should be consuming more of, whereas 'kids' foods' are associated with processed, high-sugar, low-nutrient edibles. The paper further reveals how 'kids' food' functions as an object or technology of identification for children enacted through a set of characteristics that the edibles share. Children's classification of food also reveals their savvy awareness that both 'kids' food' and 'adult food' can contain transgressive elements.
\end{abstract}

\section{Introduction}

The childhood obesity epidemic has fueled much of the recent scholarly and policy interest in food marketing to children. Over the past few decades, rates of overweight/obesity in children have steadily increased (Wang and Lobstein, 2006; De Onis and Lobstein, 2010). Significant excess body weight affects over 25 per cent of children in developed economies (Hawkes and Lobstein, 2011)—a problem that has pulled the food industry and its marketing practices into the spotlight.

In 2010, the World Health Organization released its Set of Recommendations on the Marketing of Food and Non-alcoholic Beverages to Children, seeking to "reduce the impact on children of marketing of foods high in saturated fats, trans-fatty acids, free sugars, or salt" (WHO, 2010). Intended to "guide efforts by Member States in designing...or strengthening existing policies on food marketing communications to children" (WHO, 2010:7), the report stands as one of the more mild narratives pertaining to child-oriented food marketing. So too is the US report Solving the Problem of Childhood Obesity within a Generation (2010), which frames food marketing as part of a larger need to "empower parents and caregivers" with clear nutrition messages,

\footnotetext{
${ }^{1}$ Note that I am referring to the narrative, with phrases like "guide efforts" and "reduce the impact," rather than the recommendations themselves, which are comprehensive.
} 
food/menu labels and reduced promotions of unhealthy foods (Barnes, 2010). In this report, proper food communication in the form of improved food packaging and labelling can work to encourage both the purchase of healthy products and healthy eating (Barnes, 2010:28). In contrast, scholarly articles (often in health journals) are typically more critical, pointing to a "crisis in the marketplace" (Harris et al., 2009) or "toxic environment" due to food marketing (Brownell, 2005). Some condemn the "cynical, manipulative methods" that the food industry employs to sell to children, claiming it is "time to get angry" and "take action” (Lobstein, 2009:882).

Yet, these discussions - whether over the precise nutrient profile comprising unhealthy foods or the devious marketing practices used to beguile the inexperienced-tend to overlook how children make sense of the food messages specifically targeted at them. Certainly, there has been increased interest in children's agency and how they "go about the activity of consuming" (Cook, 2010), as well as some inquiry into how children regard their own experiences with snacking (Marshall, 2010), packaged foodstuffs (Elliott, 2009a) and food culture in school settings (Ross, 1995; Stewart et al., 2006; Ludvigsen and Scott, 2009) —or even how children apply nutritional knowledge and reasoning (Harrison, 2005). But specific analyses on how children classify food in the context of today's food environment are rare. ${ }^{2}$ And although researchers have measured how child-oriented marketing or fast food logos have influenced children's taste and snack preferences (Robinson et al., 2007; Roberto et al., 2010), these are more causeand-effect studies. ${ }^{3}$ Missing is an understanding of how child-oriented food marketing has reoriented children's perceptions of what food means and the kinds of foods that are "for them”.

This article seeks to explore children's perspectives on food classification and, specifically, their thoughts about 'kids' food' and 'adult food'. The question of 'kids' food' is fascinating, because (unlike meals and festive eating occasions) the category of kids' food has been entirely constructed through advertising and marketing. Particular foods have been promoted to, and for, children — and recent scholarship points to the way children's food marketing persuades children "to eat particular foods, not on the basis of their tastiness, or other benefits, but because of their place in a social matrix of meaning" (Schor and Ford, 2007:16). Some scholars frame children's food as branded "junk" food, valued by children because it is symbolically framed as "cool" (Schor and Ford, 2007); others assert that children's fare extends beyond the narrow category of "junk" and pivots on the thematic of "fun" (Elliott, 2009b, 2010). Regardless, there is limited understanding as to the take-up of these messages by children themselves. (Even the marketing terms "pester power" or the "nag factor"-in which children pester their parents to buy particular products due to advertising/marketing messages_-provide meagre assistance,

\footnotetext{
${ }^{2}$ As an exception, see Roos, 2002

${ }^{3}$ That is, children are asked to choose between, and indicate taste preferences for, foods in branded versus non-branded wrappings.
} 
as they refer to pestering for individual products and do not translate across entire categories of goods.)

By asking children "what is kids' food and what does it mean to you?" as well as by probing their perspectives of 'adult foods', the research reveals the broader implications of particular food marketing strategies. Children view food "for them" as categorically different from regular or adult food. Examining food communication-particularly children's categorization of food-brings a deeper level of understanding to the issue of food marketing to children. It reveals the complex layers of meaning attributed to broad, binary categories (i.e., kids' food/adult food), and suggests the degree to which particular foodstuffs_ or their component parts—have become bound up with children's identities.

\section{Methods}

Focus group research is designed to help understand what people think and why; as such, it provides an ideal research method for exploratory work on children's perspectives on food and its meaning (Deacon et al., 1999; Heary and Hennessy, 2002; Morgan et al., 2002). Focus groups are recognized as a "valuable means of eliciting children's views" (Heary and Hennessy, 2002:67), because they frame the participants as experts. When kept small, the focus group helps to encourage input from shyer individuals (2002:68).

Participants were school-aged children, aged 6 to 11 years, recruited from three major urban centres across Canada (Calgary, Alberta; Ottawa, Ontario; and Fredericton, New Brunswick). These sites were selected so as to provide regional representation from Western, Central and Eastern Canada. A total of 225 children participated in small focus groups-four to six children in each group-where a moderator (using a customized moderator's guide) led the discussion. Questions probed children's food preferences and how they categorize different types of food, as well as their perspectives on food, food packaging/products, and nutrition. ${ }^{4}$

The groups were divided so that separate focus groups were held for girls and for boys; these groups were further segmented along school grades: grades $1 / 2$ (approximately 6-7 years old), grades $3 / 4$ (approximately $8-9$ years old) and grades 5/6 (approximately 1011 years old). This allowed the researchers to note differences in perspective according to both gender and age. Children's answers were digitally recorded and subsequently transcribed verbatim (with pseudonyms used for all participants); field notes were also recorded by the researchers during and after each session. A provisional list of codes was created from the conceptual framework, refined into salient themes and examined.

\footnotetext{
${ }^{4}$ Ethics approval and written parental consent, as well as written consent from the children, were obtained prior to holding the focus groups. Note that two "sets" of focus groups were held in each location for each age group/sex. One group had children drawn from "higher" education families (i.e., parent(s) with college or university degrees), and one group had children drawn from "lower" education families (i.e., parent(s) with high school). These criteria was selected because a strict look at income, particularly in light of the recent economic downturn, is less reflective of taste preferences than educational background.
} 
Iterative comparison and inductive coding techniques were used, following a grounded theory approach (Strauss and Corbin, 1998; Crabtree and Miller, 1999). This analytic strategy intends to unveil children's perspectives on food categorization and the degree of thematic consistency — or divergence-in children's discourses around the (presumably) nebulous categories of kid's food and adult food.

\section{The rise of children's food}

The concept of 'kids' food' is compelling for several reasons. First, this category of food connects with an age and/or an identity rather than a defined eating occasion (such as breakfast, lunch and snack). 'Kids' food' is not, per se, tied to ceremonial occasions (like a birthday cake) or ritual occasions (such as thanksgiving turkey), although children did discuss personalized eating rituals pertaining to foods that they consider specifically for them. Second, and more importantly, 'kids' food' is an arbitrary and relatively recent category of food. Indeed, there is no nutritional reason why discrete foodstuffs should be classified for children and others for adults. Generally, as one ages, there is a refinement of the palate and increased willingness to sample new tastes, but from a nutritional perspective, there is no reason for children to understand an apple, steak or packaged food as uniquely earmarked for a child or an adult. The reason is cultural, and recent, and powerfully driven by marketers and the food industry.

Some 90 years ago, Quaker Oats Co. launched an advertising campaign that suggested that children, cereal and confectionary should be linked; ${ }^{5}$ this connection was solidified through the 1950s and 1960s, when cereals like Sugar Crisp, Sugar Corn Pops, Corn-Fetti and Sugar Smacks became popular on grocery store shelves. It took another three decades for marketers to start advocating for even more "kidized" packaging to meet the unique needs of children (McNeal, 1999:88), and by the early 2000s, a clear process of decerealization of the supermarket was clearly evident (Elliott, 2008). Today, hundreds of child-oriented foods can be found throughout the supermarket and in every category of foods-not just in the cereal aisle.

\section{“When you think of kids' food, what do you think of?”: Themes of junk, sugar, cereal and fun}

Asking the focus groups "When you think of kids' food, what do you think of?" revealed the general consistency (across age, sex and location) of children's understanding of kids' foods. The most prevalent answer was that 'kids' food' was junk food or some variant of sugar, candy, cookie or gummy. Many children explicitly used the term "junk" or defined kids' food in terms of having the key component of sugar.

Moderator: When you think of kids' food, what do you think of? Why?

\footnotetext{
${ }^{5}$ In a 1928 issue of Chatelaine magazine, Quaker Oats ran an advertisement for Puffed Wheat and Puffed Rice, which asserted that "The WAY...to make CHILDREN like CEREAL" is to correlate it with confections. According to the advert, these puffed cereals "have a flavor enticing and delicious that children revel in them like confections” (Elliott, 2010).
} 
- Junk food! (Girl, Grade 1 [G1])

- Junk! (Boy, Grade 1 [B1])

- I think sugary stuff...cause lots of kids like sugar. (G1)

- Um, it makes me think of candy. (G3)

- Candy and chocolate because they are unhealthy and no good. (B1)

- What comes to my mind is junk food. Well, it's not just for kids but kids usually enjoy it because it's sugar and kids love sugar. (G3)

- Cookies and candies and that kind of thing. It's food that kids appreciate. It's better. ${ }^{6}(\mathrm{~B} 3)$

The identification of 'kids' food' with junk and sugar/ candy echoes earlier research that examined how children used "kets" (a particular type of sweet, usually cheaper and unwrapped-like a penny candy) to establish their separateness from adult culture. Kets "are the antithesis of the adult conception of 'real' food" (James, 1998), which is precisely what the children in the focus group gave voice to when they used terms like 'junk” and emphasized sugar.

Junk and sugar no longer reside solely in the category of confections, however, as evidenced by the second most common answer for 'kids' food': cereal. Cereals, mentioned most often by the youngest children, were, with rare exception, popular sugared cereals (again foregrounding the component of sugar) and were also recognized for their cartoon appeals.

Moderator: When you think of kids' food, what do you think of? Why?

- Lucky Charms because kids like, um, sugar. (G1)

- All of these great cereals...because it's all like sugary! (G3)

- I don't know...I think some foods are made for kids... Lucky Charms, Froot Loops, Alpha Bits...because they have, um, pictures of characters on them. (G5)

Moderator: That's really interesting. Carole, did you want to say something?

- Yes. For me, it's the same thing. And just like the characters like Froot Loops, Lucky Charms or Alpha Bits-yeah, like the kids see that and say 'oh mom, I like those!' (G5)

Importantly, it was not merely that the cereals were considered 'breakfast candy' by children (with the idea of confection extending into meals and the foods that define those

\footnotetext{
${ }^{6}$ In light of recent initiatives to ban the sale of sugary sodas in schools (and proposals for implementing soda taxes), it is interesting that in their discussion of "junk" and "sugar," not one child identified 'soda pop' as a particular kids' food. Soda pop is a beverage, not a food; but when asked about 'adult food', many children identified beverages such as coffee or alcohol.
} 
meals). Cartoon characters, be it industry-created 'mascots' (such as Lucky the Leprechaun) or cross merchandising (such as Dora the Explorer or Transformers), were also mentioned as core features of 'kids' food'-regardless of the sugar content. A girl in Grade 4 explained how one might tell the difference.

- They don't have pictures of actually real things, but they actually have their own mascots to them that...that aren't actually real...'cause if you look, they're cartoons.... (G4)

The third most common thematic that children associated 'kids' food' with was concrete/descriptive dimensions (Young, 2000:504), that is, descriptors that deal with perceptual differences of food, such as its shape, size or colour.

- Lucky Charms because kids like the little shapes. (G1)

- Cookies that are shaped... and some popsicles that are shaped. I've seen a bunch of them at the store, but my parents don't let me have them. (G5)

- Ritz crackers...um sometimes they come in little red packages and they're like cheese and they're smaller....Yeah, well, they're kind of like bite size. (G3)

- Well, I think that kids' foods are like stuff that you see on TV, like pretty much comic characters. I mean like the Dora [labelled food product] would be good for little kids, and the Mini Chefs ${ }^{7}$... and you just think of mini and you think of kids. Kids' food would mean food that you kind of either have more sugar in them or be like Mini Chefs that have the animal shapes and stuff. (B3)

Different colours and fun shapes are, in the children's perspectives, unique to kids' food, because not one mention was made of colour or shape in their discussion of adult food. Size, when mentioned, was always in the context of something small. (This is consistent with other studies on children's accounts of snacking, where the snack is a "mini meal" or a small thing [Marshall, 2010].) In the case of kids' food, it is the edible itself that is small rather than the size being mandated by the eating occasion of the snack.

In short, notions of junk/sugar, cereal, unusual colours/ shapes and small sizes represent the most salient themes from children's thoughts on 'kids' food'. Other emergent ideas generally supported concepts related to the dominant themes. A small number of children (equally distributed across age/sex lines) specifically explained that kids' food was "fun", also pointing to examples that illustrate descriptive dimensions - animal-shaped cookies or brightly coloured fruit snacks that gush or can be rolled out and stretched. For one girl, even non-packaged, healthy foods could be fun but required some additions in order to fit this criterion. Her helpful advice pertained to adorning food to make it "more complicated"-but the discussion is really about heightening the perception of fun.

\footnotetext{
${ }^{7}$ President's Choice Mini Chefs is a popular Canadian food brand targeted at children.
} 
- Maybe some kids...it's mostly little kids...don't like stuff that's healthy like vegetables and stuff. But when you make it look fun, then they'll probably eat it more often.... You can add stuff onto like vegetables and stuff like cheese or sauce or something like that...so it's more complicated. (G3)

\section{Moderator: Complicated?}

- Well, there's lots of designs and stuff [on kids' food]. This sentiment was echoed by another girl in same group.

- I was looking at this book and it has like vegetables in it, but they're not just plain vegetables...like they have shapes so they look more appetizing. So, like, if they look nice, then they'll eat it, but if they don't look nice, then they won't eat it. Like, um...they use peas for the eyes and for the mouth, and they kind of cut them into faces. (G3)

Shapes, colours and interactivity contribute to the 'fun' in kids' food. Other children treated the concept of fun as self-explanatory ("It's kids' food because it's fun.").

"Unhealthy" was a term used by a small number of participants, although this concept simply extends the notion of junk, underscoring the ways that children recognize kids' food in opposition to other food. Here, participants used distinguishing phrases that represent functional dimensions of food (Young, 2000:504). Functional dimensions distinguish one food from another (including food origins, or health dimensions such as "good for you/healthy" or "bad for you/ not healthy").

Moderator: When you think of kids' food, what do you think of? Why?

- Candy and chocolate because they're unhealthy and no good. (B1)

- Something that your parents don't want you to eat. (B3)

Young (2000:506) has found that "the healthy/unhealthy constitutes a basic dimension children use when classifying foods". What proves significant is that the children who applied this dimension in the focus groups used it to distinguish food for particular audiences - that is, 'kids' food' (unhealthy/undesirable) versus 'adult food' (healthy/ desirable). Kids' food thus becomes part of a discourse about identity (Mechling, 2000:18) constructed in opposition to parent desires.

Given the vibrant, current policy discussion pertaining to food advertising, one notable finding is that branded food products were rarely mentioned in children's discussion of 'kids' food', with the exception of the names of sugary cereals. McDonald's was mentioned by only two children (in different focus groups), although some children revealed keen awareness of cross merchandising on packaged foods, such as Dora the Explorer, Elmo, or Transformers. One girl in Grade 1 observed the following: 
- I think of...little boxes that have, like, kids' designs. Things that kids like. I know, like yah, when I was a kid I liked Sesame Street or Barney, but like nowadays they have... they always have things to attract kids' attention because of the characters and stuff. (G1)

Instead of referencing brands like McDonald's, children identified particular kinds of food - those often associated with fast food restaurants and 'kids' fare'. Chicken nuggets, pizza, hotdogs and/or hamburgers were all named as 'kids' food' by a small number of children - all edibles were designed to be eaten with one's hands. These foods, like 'fun foods', are more interactive and informal than the more 'serious' adult fare that children subsequently discussed.

Generally, children framed 'kids' food' as either explicitly or implicitly oppositional, highlighting its characteristic features as junk or sugary-or they drew attention to perceptual characteristics or interactive qualities that made food more about engagement, play or boundary breaking (i.e., no need for utensils). As one child observed, "kids like to eat stuff with their hands and adults are more, like, mannered and they use more knives and forks for more things" (G5). One moderately diverging discourse emerged as the fourth most prevalent theme, in which children identified fruits or vegetables as kids' food. It can be considered moderately diverging because, as noted earlier, some of these mentions were in the context of "dressing up" the vegetables to make them look fun. Other children recognized that a particular authority resided behind their selection“fruit, um, 'cause you're supposed to eat a lot of fruit” (B5) — making it clear that the classification did not come from them. Finally, some children mentioned fruits and vegetables as kids' food but could not provide any explanation as to why.

\section{"What kinds of foods are adult foods?": Contrasts in stimulation, classification and value}

Children's perceptions of 'kids' food' (described earlier) might strike some as obvious or not particularly illuminating. The real purchase in this study emerges when framing participants' discussions in light of their thoughts on adult fare. When subsequently asked, "What kinds of foods are adult foods?" and probed on why, fascinating contrasts in classification and value materialize. Unlike the children's identification with junk food, sugar, candy and (sugary) cereal, the top 'food groups' identified as 'adult foods' could be classified under the headings of fruits/vegetables or protein. Under the fruits/vegetables category, salad is frequently mentioned; associations are also sometimes made with health and responsibility.

Moderator: When you think of adult food, what do you think of? What kinds of foods are adult foods?

- Brussel sprouts or asparagus. (B5)

- Something healthy...apples, bananas...steak. (B3)

- Healthy and stuff like vegetables. (G3) 
- Um...the things that adults don't want to eat that are healthy they have to eat to be a good role model for their children and people around them...like if they don't want to eat peas or something...they'll still eat them to be a good role model.... (G3)

Protein sources are identified as 'adult food' just as frequently as fruits and vegetables/salad. Children mentioned chicken, beef, steak or fish; the participants from Fredericton, New Brunswick, often identify lobster and shrimp as distinctly adult. Presumably, meat speaks for itself, because the reasoning behind what makes protein 'adult food' is frequently not provided. Yet, the key themes pertaining to adult food generally echo those already discussed in the context of 'kids' food'-albeit on the other end of the spectrum. Thus, in contrast to the 'junk' characterizing 'kids' food', there are references to health. Similarly, the recognition of "something small" for children gives way to the opposition of larger sizes for adults, even if it is not calorific.

- I think of steak or something big. (G5)

- It's bigger food than kids' [food]. (B3)

- When I think of adult food, I think more of stuff that kids can't have...like a big bowl of soup compared to a little bowl of soup. (G3)

- A lot and a lot of salad. (G1)

Concrete/descriptive dimensions, which children used to describe kids' food, were not prevalent in the discussion of adult food (with the previously noted exception of larger size). When raised, the idea was that adult food was plain or lacking elements.

- Something just came to my mind... adult food is boring food. (B3)

- Anything with no fat, no sugar and no crap like that. (G4)

- More like diet food because some of them diet a lot. Sometimes. (B5)

- Usually my dad will eat Cheerios, and probably eat plain food. Usually, the cereal he eats doesn't really have any colour, it is just brown. He'll eat big sandwiches.... (G3)

This plainness stands in sharp contrast to the narratives suggesting that 'kids' food' is fun. Adult food is plain - but some children also observe characteristics of spiciness in adult fare. This is not necessarily a conflicting discourse in terms of plainness: what 'spices up' children's food is colour and shape, whereas the plainness of adult food stems from its lack of colour/sugar/shape. 'Spice' in adult food instead pertains to its taste profile.

- Well, since kids have more sensitive taste, it [adult food] would have more spices and they would have...less fat, less sugar... which takes out all the fun. (G1)

- I think of spicy foods. It has, like, a stronger flavor and taste. (G4) 
One overarching and significant finding is that children implicitly seem to recognize 'kids' food' as packaged and highly processed food, whereas "plain" adult fare is not out of a box, not packaged and largely unprocessed food.

- [Adult food] doesn't have to necessarily have to be not bad for you, but they usually don't go out buying like Pop Tarts and stuff. Um, they just like different tastes. (G5)

- Some foods are adult foods and don't have boxes. Like when you go to the store and you buy carrots, you have to get a bag for it. (G1)

A final finding pertains to the theme of stimulation. It is important to note that the most prevalent theme that emerged around 'adult food' was that of stimulants—namely, wine, beer, alcohol, caffeine and drugs. I raise this point last because although stimulants were mentioned with only slightly more frequency than fruits/vegetables and protein, they are not actually foods. Boys, and particularly those in the highest grades, were most likely to observe that wine, beer or alcohol were adult foods; the older boys were also the most likely to mention coffee and energy drinks as uniquely adult. (The significance of this finding will be discussed shortly.)

\section{The Kids' Food/Adult Food Divide: Discussion and Implications}

Exploring children's perspectives on 'kids' food' and 'adult food' is a seemingly small question with large implications. Literature in the sociology of childhood has long observed that childhood is defined in opposition to adulthood (James and James, 2008:23-27), and it is not surprising that children internalize and give voice to these oppositions. What is new is the degree to which the adult/child divide now expresses itself in terms of how children categorize food—and not just particular snacking occasions (Marshall, 2010), individual products, or categories like confectionary (James, 1998). The categorization is far broader, pertaining to food itself. Mechling remarks that "the consumption of food can be part of the performance of identity" and that children use food "to mark the distinction between child and adult" (2000:18). Ludvigsen and Scott's research on "what food means to children" similarly observes a "socially constructed distinction between children's food and adults' food” (2009:426). This present study supports these observations and brings new insight into how food marketing appears to have influenced children's classification of food in a more general sense. When children think of 'kids' food', they generally think of junk food, sugar, sugary cereals and the fun shapes and unusual colours characterizing much of contemporary child-oriented packaged food. When children think of 'adult food,' they think of fruits, vegetables and meat. Here, I do mean children, painting the perspectives with fairly broad strokes - and there is a particular reason why. As detailed earlier, the research design was created to track differences in perspectives in terms of age and sex. Although there were clear differences with regard to the children's perspectives in terms of age and sex for the other questions posed throughout the focus group, for the specific questions pertaining to food classification (i.e., what is kids' food/adult food), the responses were consistent, regardless of age and gender. This is why 'differences' were 
not teased out, because they were not clearly discernable in the children's perspectives on food classification.

What is particularly troubling about how the children classified foods is that 'adult foods,' to them, are generally the unprocessed fruits, vegetables and meats that all North Americans should be consuming more of, whereas 'kids' foods' are associated with processed, high-sugar, low-nutrient edibles. This point must not be glossed over. At a time where childhood obesity is considered an epidemic and where inadequate fruit and vegetable consumption has been deemed "an important public health issue" (Shields, 2004), it is troubling that children classify unprocessed fruits and vegetables as "for others" and processed "junk" as "for them". In fairness (and recalling the critiques mentioned at the onset of this article pertaining to the "toxic environment" due to food marketing), the responsibility for this classification does not lie entirely with the food industry. Recent bestselling cookbooks such as The Sneaky Chef: Simple Strategies for Hiding Healthy Foods in Kids' Favorite Meals (2007) and Deceptively Delicious: Simple Secrets to Get Your Kids Eating Good Food (2007) pivot on the premise that fruits and vegetables must be snuck into recipes in order to make children eat them. As The Sneaky Chef explains "[y]our kids will never suspect that there's blueberries pureed into their brownies, cauliflower in their mac ' $n$ ' cheese, or sweet potatoes in their lasagna-but they'll love every bite” (Lapine, 2007). Deceptively Delicious mirrors this sentiment; its author expresses her joy at tricking her children into eating vegetables "and other healthful additions" without their knowledge (Seinfeld, 2007:11) and calls this "loving deception" (2007:13). These cookbooks communicate the message that children could not possibly eat fruits and vegetables on purpose_only by accident. Equally, the cookbooks communicate that savvy parents feed children food that they already like (pleasure is not about new tastes) and that fun is central to food. The Sneaky Chef asserts that:

[Some food]...can be bad for one's health. The age-old problem is that if we eliminated the 'bad' foods and kept only the 'good' ones, eating might not be fun anymore. Adults continuously grapple with this almost existential dilemma.... (Lapine, 2007:15)

Setting aside the flagrant misuse of existentialism, the worry that "eating might not be fun anymore" is not, in fact, an age-old problem. Evaluating food in light of its fun factor, as well as fretting that it might not be fun, is in fact very recent. Indeed, one might fairly ask the question "why does food have to be fun?" Numerous implications arise from marketing food as eatertainment to children. ${ }^{8}$ It is worth noting that it is only in the world of children's food that edibles are valued for their capacity to entertain.

\footnotetext{
${ }^{8}$ For a detailed discussion on the implications of marketing food as eatertainment to children-as well as why this type of marketing has managed to gain traction with children (and their parents) - see Elliott (2010, 2009b).
} 
For the children in the focus groups, the articulation of 'adult food' as fruit, vegetables and meat, and of 'kids' food' as junk, sugary cereal and edibles with unique shapes/ colours, also came with more implicit assumptions around questions of health. Healthy is for adults, and 'junk' is for kids ${ }^{9}$ - and it is worth noting that four children specifically said that 'kids' food' is "unhealthy", whereas none of the participants deemed 'adult food' unhealthy. Even though some children observed that there were clear problems with this trend, the question of 'fun' in 'kids' food' seemed to win out.

- Well, kids' food should be, at least, have a little bit of vitamins or iron in them...they should, but they don't... they don't really focus on the kids' health...they usually focus on what the kids will want to buy so that they're going to focus on Elmo, or Dora, or Transformers or stuff like that. (G1)

Indeed, the way that children described the characteristics of kids' food is more significant than the type of food: 'kids' food' is sugary or junk, and it is fun. The theme that 'kids' food' is fun is described in many different ways, with reference to colours and shapes, pictures and cartoon characters. "Some packages...are really bright”, observed one boy (G1), whereas two girls in the grades 3-4 focus groups also recognized that 'kids' food' had to do with the visual, not taste:

- Um, stuff that's like colourful and all different shapes and something that would attract you to look at it and buy it. (G3)

- I sort of agree with Eliza...like colourful and it has cool shapes and stuff. (G3)

Such emphasis on the characteristics of kids' food also provides insight into why specific brands were rarely mentioned. Recent research by McAlister and Cornwell suggests that very young children (i.e., preschoolers) recognize the brands "with which they have the most experience" (2010:221), that is, fast food and grocery store brands (2010:208, 221). Part of what makes these brands salient, the authors suggest, are the attractive "colors, shapes, or characters used to define the image" (2010:221). This study reveals the degree to which the broader category of fun (characterized by colours, shapes, characters, etc.) eclipses the smaller components of brand names. Brands are less significant than the characteristics of fun (or health or boringness, etc.) and what makes these foods fun (etc.) to children. Fun is relevant (and salient) in the lives of young children. Although health is certainly a part of the discourse when it comes to food - children seem quite familiar with themes pertaining to health-it is not prioritized as per the discussions around fun. (Note that cookbooks like The Sneaky Chef and Deceptively Delicious both suggest that the health qualities are salient to parents but not to children; they pivot on the premise that the health qualities of foods must not only be downplayed but in fact also hidden from children.)

\footnotetext{
${ }^{9}$ As previously noted, this did not hold true for all respondents. Some children identified kids' food as "healthy food", but it was not a dominant perspective.
} 
Thus (as previously noted), what 'spices' up 'kids' food' for children is fun, which pertains to both the interactive and surface aspects of food. Spice in adult fare, in contrast, refers to the taste profile of the food; it resides in the food itself. Thus, one is left with a series of oppositions: spice for adults/ fun for kids, health for adults/junk for kids, and unprocessed edibles/processed foodstuffs. Even the notion of sugar as a desired element by children in children's food contrasts with the concept of lack (of calories, sugar, fat or salt) in adult fare. In this fashion, the concept of 'kids' food' acts as an object or technology of identification ${ }^{10}$ for children-enacted not through a singular product or meal occasion but through a general set of characteristics that the edibles share. This collective sense making, this classification of 'kids' food', is bolstered by its polarity to 'adult food.'

These oppositions do not mean that the children are destined for unhealthy or unnatural eating, however. It is true that children were consistent in identifying what they saw as the defining characteristics of 'kids' food', which is, as I stated, a technology of identification for children. Yet, over 10 per cent of the children stated that their parents never or "barely" purchase 'kids' food', ${ }^{11}$ and an emerging (yet understated) theme articulated primarily by the older girls was the recognition of 'kids' food' as a treat. The point is this: the fact that children both recognize and can articulate the characteristics of 'kids' food' does not, by definition, make them adverse to the idea of eating fruit or vegetables or meat. 'Kids' food' serves a communicative function, not just a (poorly) nutritious one, and the question of fun or play as part of what 'kids' food' means is centrally important.

Overall, children's classification of food proves to be remarkably sophisticated. Their answers trouble perspectives that seek to document direct "stimulus-response" patterns (i.e., responses to specific advertisements or brand appeals) because the children's answers speak more generally to characteristics and classifications and what kids' food has to offer than to a particular food item or brand. That the children interviewed were equally able to detail the characteristics typifying 'adult food' reveals their awareness of food "for others" as well as food "for themselves". Within the oppositions articulated, children also perceptively observed that transgressive elements are to be found in both

\footnotetext{
${ }^{10}$ Framing kids' food as an object of identification or a technology of identification means looking at the material artefact of kids' food and the way that (i) it has come to communicate very particular meanings to children and (ii) it has become a marker of identity formation. Children both identify with these foods and use them to mark identity. This article has dealt with the different perceptual characteristics of kids' food, its concrete/descriptive dimensions (i.e., size, shape and colour), its functional dimensions and the way that the particular audience of the child is imagined through kids' food's appeal to, and insistence on, fun. All of these features combine for one to understand fun food as a technology of identification. As noted, kids' food as a technology of identification is enacted not through a singular product or meal occasion but through a general set of characteristics that the edibles share.

${ }^{11}$ At the conclusion of the focus groups, we asked the children whether their parents ever purchased them kids' food. This was a specific "yes/no" type of question (although some of the children responded with "sometimes", "barely", "never", etc.). Although focus group research does not typically quantify responses, the "yes/no" format of this particular question does allow for quantification (i.e., over $10 \%$ of the children stated that their parents never or "barely" "purchase 'kids' food').
} 
kids' and adult food. Consider that the most prevalent theme for 'kids' food' was junk/sugar; the most prevalent answer for 'adult food' was, in fact, wine/beer/alcohol/coffee. All are stimulants. Sugar is for kids, and alcohol/caffeine is for adults. One boy observed that sugar "makes you hyper" (G1), whereas two of the older girls expressed the meaning of these stimulants in a system of comparisons and contrasts.

- Most parents like...alcohol better than sugar, so kids' foods usually have like lots of sugar and something that they like instead of alcohol, which really isn't good for kids.... (G5)

- ...kids [who] eat a lot of candy can...get addicted, so it's like beer for kids. (G5)

Equating sugary food for children with alcohol for adults implies that these children see both as not good for you. Transgressive elements or "should not's" are thus present in both categories of food, both 'kids' food' and 'adult food.' Children recognize such transgression as part of the appeal. So while 'kids' food' is understood as junky and fun, the category of 'adult food' (although plain and healthy) also has its equivalent of 'junk'.

By exploring children's perspectives on 'kids' food' and 'adult food', we move beyond the typical narrow focus on the nutrient profiles of specific advertised products to engage with the social significance of what it means to market foods in a particular way. The symbolic marketing of children's foods raises fascinating questions about how children classify food generally and about what they consider to be uniquely "for them" and "for others". Special attention should be placed on the values underpinning children's classifications of food: 'kids' food' as desirable and oppositional (junk and fun, and processed) and "adult food" as healthy and spicy-yet-boring, and unprocessed. Questions of classification work to illuminate what symbolic marketing has really meant to children and suggest a new avenue of inquiry into children's development and understanding of preference.

\section{Acknowledgements}

Funding for this research was generously provided by the Canadian Institutes of Health Research (FRN 86633). The author would like to thank Shelley Elliott for her excellent work in coding, and to research assistants Meghan Brierley, Brenda McDermitt and Lauren Levesque for their contributions in organizing and helping to conduct the focus groups. Thanks to Glenn Dixon for his comments on a previous draft of the paper, and to the anonymous reviewers and David Marshall for a range of thoughtful suggestions and recommendations.

\section{References}

Barnes M. 2010. Solving the Problem of Childhood Obesity within a Generation. White House Task Force on Childhood Obesity Report to the President. Washington, DC. 
Brownell K. 2005. Does a 'toxic' environment make obesity inevitable? Obesity Management 1(2): 52-55.

Cook DT. 2010. (Re)Placing Mothers: Rethinking Child Consumption in Light of Contemporary Motherhoods. Paper presented at the 4th Child and Teen Consumption Conference, Department of Child Studies, Linköping University, June 21-23, Campus Norrköping, Sweden.

Crabtree BF, Miller WL. 1999. Doing Qualitative Research, 2nd edn. Sage Publications: Thousand Oaks, CA.

De Onis M, Lobstein T. 2010. Defining obesity risk status in the general childhood population: which cut-offs should we use? International Journal of Pediatric Obesity 5: 458-460.

Deacon D, Pickering M, Golding P, Murdock G. 1999. Researching Communications: A Practical Guide to Methods in Media and Cultural Analysis. Arnold: London.

Elliott C. 2008. Marketing fun foods: a profile and analysis of supermarket food messages targeted at children. Canadian Public Policy 34(2): 259-274.

Elliott C. 2009a. 'Healthy food looks serious': how children interpret packaged food products. Canadian Journal of Communication 34(3): 359-380.

Elliott C. 2009b. Entertaining eats: children's 'fun food' and the transformation of the domestic foodscape. Material Culture Review 70: 34-43.

Elliott C. 2010. Eatertainment and the (re)classification of children's food. Food, Culture and Society 13(4): 539-553.

Harris JL, Pomeranz JL, Lobstein T, Brownell KD. 2009. A crisis in the marketplace: how food marketing contributes to childhood obesity and what can be done. Annual Review of Public Health 30: 211-225.

Harrison K. 2005. Is ‘fat free’ good for me? A panel study of television viewing and children's nutritional knowledge and reasoning. Health Communication 17(2): 117-132.

Hawkes C, Lobstein T. 2011. Regulating the commercial promotion of food to children: a survey of actions worldwide. International Journal of Pediatric Obesity 6(2): 83-94.

Heary C, Hennessy E. 2002. The use of focus group interviews in pediatric health care research. Journal of Pediatric Psychology 27(1): 47-57.

James A. 1998. Confections, concoctions, and conceptions. In The Children's Culture Reader, Jenkins H (ed). The New York UP: New York; 394-403.

James A, James A. 2008. Key Concepts in Childhood Studies. Sage: London.

Lapine MC. 2007. The Sneaky Chef: Simple Strategies for Hiding Healthy Foods in Kids’ Favourite Meals. Running Press: Philadelphia, PA. 
Lobstein T. 2009. Marketing of unhealthy food to young children: time to get angry, get active. Public Health Nutrition 12(6): 882.

Ludvigsen A, Scott S. 2009. Real kids don’t eat quiche. Food, Culture and Society 12(4): 418436.

Marshall D. 2010. What's in the Cookie Jar: Young Children's Accounts of Snacking. Paper presented at the ANR Colloque International Consommations Alimentaires, Cultures Enfantines et Education Angouleme, April 1-2, France.

McAlister AR, Cornwell TB. 2010. Children’s brand symbolism understanding: links to theory of mind and executive functioning. Psychology and Marketing 27(3): 203-228.

McNeal J. 1999. The Kids Market: Myths and Realities. Paramount Market Publishing: Ithaca, NY.

Mechling J. 2000. Don’t play with your food. Children’s Folklore Review 23(1): 7-24.

Morgan M, Gibbs S, Maxwell K, Britten N. 2002. Hearing children’s voices: methodological issues in conducting focus groups with children aged 7-11 years. Qualitative Research 2(1): 520.

Roberto CA, Baik J, Harris JL, Brownell KD. 2010. Influence of licensed characters on children's taste and snack preferences. Pediatrics 126(1): 88-93.

Robinson TN, Borzekowski DL, Matheson DM, Kraemer HC. 2007. Effects of fast food branding on young children's taste preferences. Archives of Pediatrics \& Adolescent Medicine 161 (8): 792-797.

Roos G. 2002. Our bodies are made of pizza—food and embodiment among children in Kentucky. Ecology of Food and Nutrition 41(1): 1-19.

Ross S. 1995. 'Do I really have to eat that?': a qualitative study of schoolchildren's food choices and preferences. Health Education Journal 54: 312-321.

Schor J, Ford M. 2007. From tastes great to cool: children's food marketing and the rise of the symbolic. The Journal of Law, Medicine \& Ethics 35(1): 10-21.

Seinfeld J. 2007. Deceptively Delicious: Simple Secrets to Get Your Kids Eating Good Food. HarperCollins: New York.

Shields M. 2004. Nutrition: Findings from the Canadian Community Health Survey. Measured Obesity: Overweight Canadian Children and Adolescents. Statistics Canada: Ottawa, ON. Chart 9: Overweight and obesity rates, by fruit and vegetable consumption, household population aged 2 to 17, Canada excluding territories, 2004. Available at http://www.statcan.gc. ca/pub/82-620m/2005001/c-g/child-enfant/4053588-eng.htm [accessed on 19 November 2010]. 
Stewart K, Treasure E, Gill P, Chadwick B. 2006. Understandings about food among 6-11 year olds in South Wales. Food, Culture and Society 9(3): 317-336.

Strauss A, Corbin J. 1998. Basics of Qualitative Research: Techniques and Procedures for Developing Grounded Theory, 2nd edn. Sage: Thousand Oaks, CA.

Wang Y, Lobstein T. 2006. Worldwide trends in childhood overweight and obesity. International Journal of Pediatric Obesity 1: 11-25.

WHO. 2010. Set of Recommendations on the Marketing of Foods and Non-alcoholic Beverages to Children. World Health Organization: Switzerland.

Young BM. 2000. Children's categorization of foods. International Journal of Advertising 19(4): 495-508. 